\title{
A case report of Grover's disease from immunotherapy-a skin toxicity induced by inhibition of CTLA-4 but not PD-1
}

\author{
Marc Uemura ${ }^{1 \dagger}$, Faisal Fa'ak ${ }^{1 \dagger}$, Cara Haymaker ${ }^{1}$, Natalie McQuail ${ }^{1}$, Elizabeth Sirmans ${ }^{1}$, Courtney W. Hudgens ${ }^{2}$, \\ Lydia Barbara', Chantale Bernatchez ${ }^{1}$, Jonathan L. Curry ${ }^{2}$, Patrick Hwu' ${ }^{1}$, Michael T. Tetzlaff ${ }^{2}$ and Adi Diab ${ }^{1 *}$ (D)
}

\begin{abstract}
Background: Immune related adverse events (irAEs) are common side effects of checkpoint inhibitory (CPI) therapies targeting CTLA-4 and PD-1/PD-L1. Grover's disease is an uncommon dermatologic condition with unclear pathogenesis previously reported as an irAE with ipilimumab.

Case Presentation: We report an additional case of ipilimumab-induced Grover's disease. Interestingly, this dermatologic side effect did not appear with use of anti-PD-1 therapy in our patient. Immune analysis was performed and suggests a possible role of Th2 cells in its patholgenesis.

Conclusion: This case suggests that Grover's disease is an irAE induced by Ipilimumab. Our immune analysis suggests that Th2 cells may be pathogenic mediators which warrants further study.
\end{abstract}

\section{Background}

Grover's Disease, or transient acantholytic dermatosis, was first described in 1970 in 6 patients presenting with an unusual pruritic, papulovesicular dermatosis [1]. Thereafter, retrospective studies described the disease's distinct clinicopathologic features. Predominantly on the trunk, back and extremities in adults over 40 years of age, the distinctive skin lesions typically present with a variety of histologic patterns (like pemphigus, Darier, and Hailey-Hailey) that mimic other acantholytic dermatoses [2, 3]. Given its rarity, the disease's precise etiology and pathogenesis remains unclear but studies have suggested numerous entities, including drugs (recombinant IL-4; RAF inhibitors), solid and hematologic malignancies, organ transplantation, ultraviolent exposure and heat [4-7]. Recently, Grover's disease was reported in a patient with metastatic melanoma treated with ipilimumab [8].

\footnotetext{
* Correspondence: adiab@mdanderson.org

This article has been updated to correct the name of Faisal Fa'ak.

${ }^{\dagger}$ Equal contributors

${ }^{1}$ Department of Melanoma Medical Oncology, University of Texas-MD

Anderson Cancer Center, Houston, TX, USA

Full list of author information is available at the end of the article
}

Here, we report another case of ipilimumab-induced Grover's disease and provide a possible immunological mechanism underlying the disease's pathogenesis in relationship to CTLA-4, but not PD-1 inhibition. To our knowledge, there are no other cases describing this immunologic mechanism for ipilimumab-induced Grover's disease in the medical literature.

\section{Case presentation}

A 73 y/o male presented with stage IIA cutaneous melanoma of the left shoulder and underwent surgical resection in 2003. In November 2012, he developed bilateral lung nodules with no other metastatic sites. A right lung biopsy at this time confirmed metastatic melanoma. He initiated therapy with ipilimumab at $3 \mathrm{mg} / \mathrm{kg}$ IV every three weeks. After his second treatment dose, he developed an intensely pruritic, papulovesicular rash over his chest, bilateral upper extremities, and back. Prior to this, the patient had no history of skin rashes. He received methylprednisolone (40 mg IV initially, then $20 \mathrm{mg}$ IV 1 month later without topical therapy) with significant pruritus improvement; however, the rash persisted. His outside physician then discontinued ipilimumab in March 2013 because of persistent rash symptoms and transitioned the patient to close observation. 
Remarkably, his lung nodules remained stable based on CT imaging performed every six months.

In July 2014, we evaluated the patient for progressive lung and liver metastases. He underwent a liver biopsy that confirmed metastatic melanoma with mutations in $B R A F$ G466A and RET W917. Interestingly, he continued to have an extensive, erythematous, papular rash over his chest, arms, and back that was asymptomatic and had persisted since his previous ipilimumab therapy. He had no other oral ulcers or skin lesions. A skin biopsy was performed and histopathologic examination revealed suprabasal acantholysis with associated overlying dyskeratosis, consistent with Grover's disease (Fig. 1a-c). The acantholysis extended to the spinous layer of the epidermis with some keratinocytes retaining partial attachment to one another. In addition, there was a superficial dermal mononuclear inflammatory infiltrate. Immunohistochemical (IHC) studies demonstrated a predominance of $\mathrm{CD} 3+\mathrm{T}$-cells with a predominance of $\mathrm{CD}^{+}$over $\mathrm{CD}^{+}$T-cells (Fig. 1d-f). Because anti-PD-1 therapy had not been FDA-approved at the time, he received systemic chemotherapy with disease progression. Considering his BRAF G466A mutation, we subsequently initiated single agent trametinib but his disease again progressed. Both chemotherapy and trametinib were initiated after the patient's repeat skin biopsy.

Thereafter, we started pembrolizumab at $2 \mathrm{mg} / \mathrm{kg}$ IV every three weeks. He tolerated therapy without significant toxicities. His previous rash symptoms remained stable during therapy with pembrolizumab. Unfortunately, after two cycles he continued to have rapid, progressive disease, and we discontinued therapy. Given the previous favorable response with ipilimumab, we re-initiated this therapy, but after one ipilimumab dose, he experienced grade 2 rash exacerbation with an intensely pruritic, papulovescular skin eruption on his upper back. A repeat biopsy confirmed Grover's disease

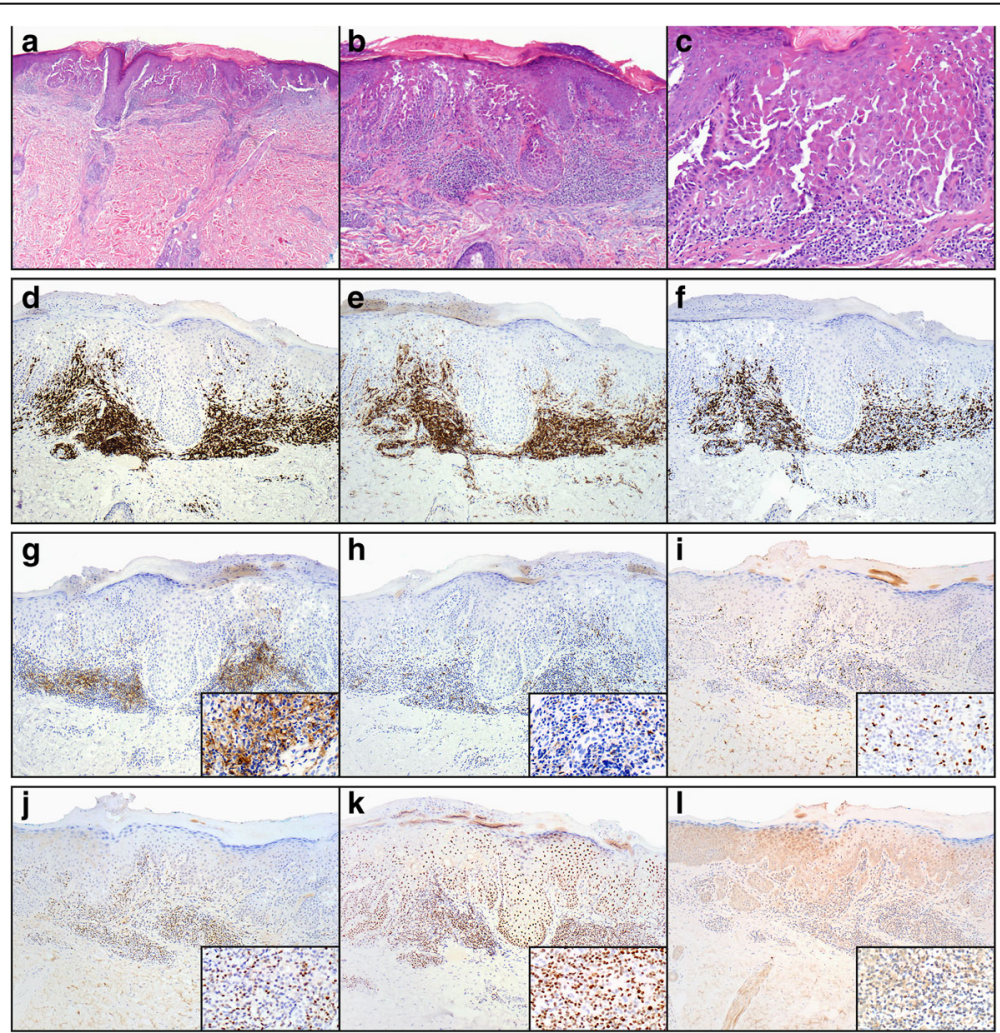

Fig. 1 Histopathologic and immunophenotypic studies of ipilimumab-induced Grover's disease. a-c Scanning magnification of skin with acantholytic dyskeratosis (a, H\&E, 20x). Higher magnification reveals skin with suprabasal acantholysis and overlying dyskeratosis; (b, H\&E, 100x) and (c, H\&E, 40x). Immunohistochemical studies demonstrate a predominance of CD3+ T-cells (d, anti-CD3, 100x) comprised of a predominance of CD4+ T-cells (e, anti-CD4, 100X) over CD8+ T-cells (f, anti-CD8, 100X). Additional immunohistochemical studies demonstrate strong expression of PD-L1 by the inflammatory cells (g, anti-PD-L1, 100x; inset, 400x). Scattered cells express PD-1 ( $\mathbf{h}$, anti-PD-1, 100x; inset 400x). There is scattered nuclear expression of FoxP3 (i, anti-FoxP3, 100x; inset 400x) and T-beta ( $\mathbf{j}$, anti-T-Bet, 100x; inset, 400x) but strong diffuse nuclear expression of Gata-3 by the majority of the inflammatory cells as well as the overlying keratinocytes (k, anti-Gata-3, 100x; inset: 400x). Antibodies for RORgT are essentially negative in the infiltrate. There is strong background staining in the skin tissue (I, anti-RORgT, 100x; inset: 400X) 


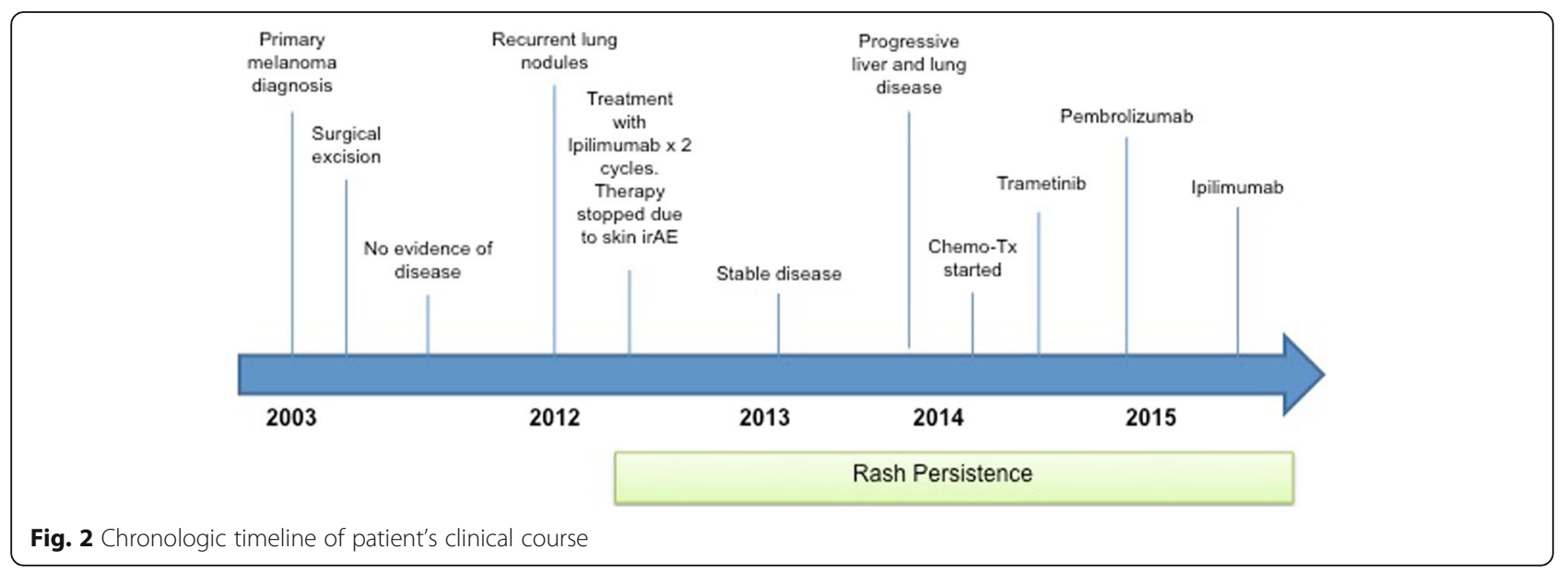

exacerbation. We started topical steroids and oral antihistamines with minimal symptom improvement. Thereafter, he received one additional ipilimumab dose but transitioned to hospice after demonstrating terminal, progressive disease. A treatment course timeline is included as Fig. 2.

\section{Discussion}

Our case illustrates another example of ipilimumabinduced Grover's disease (transient acantholytic dyskeratosis). In our patient, there was a direct relationship between onset of characteristic skin lesions with initial ipilimumab administration and lesion exacerbation after drug re-initiation. Remarkably, this immune finding does not seem to be a universal consequence of all CPI therapies as we observed stability of skin lesions with pembrolizumab, a PD-1 inhibitor. Moreover, ipilimumabinduced Grover's disease appears to be a rare phenomenon given the paucity of reported cases. Nevertheless, it is important to emphasize that the histopathologic changes seen here are indistinguishable from that seen in sporadic Grover's disease.

IrAEs are common in patients treated with ipilimumab. Phase 3 studies showed that the most common ipilimumab-associated irAEs were dermatologic, gastrointestinal, endocrine, and hepatic in nature $[9,10]$. While the pathogenesis of irAEs is unclear, CD4 ${ }^{+}$T-cells have been shown to play a significant role [11]. Specifically, $\mathrm{T}$ helper 17 cells (Th17), which are a distinct lineage of $\mathrm{CD}^{+}$T-cells that produce characteristic cytokines like IL-17 and IL-22, have been implicated in a variety of autoimmune diseases and irAEs, particularly colitis [12]. Supporting this finding, pre-clinical studies have shown that blocking the CTLA-4:B7 interaction on antigen presenting cells and $\mathrm{T}$ cells, which is the mechanistic action of ipilimumab, potentiates Th17 cell differentiation in vivo and in vitro [12]. It is thought that blocking this CTLA-4:B7 interaction with ipilimumab, therefore, can potentiate Th17 mediated autoimmunity. In fact, a recent study reported on the correlation between IL-17 up-regulation, and the development of immune related colitis in patients treated with ipilimumab [13].

In our patient, IHC tissue analyses demonstrated marked $\mathrm{CD}^{+}$lymphocyte infiltration and a predominance of $\mathrm{CD}^{+}$over $\mathrm{CD}^{+}$T-cells (Fig. 1e-f). Additionally, there was moderate expression of PD-1/PD-L1 (Fig. 1g-h). Considering the above hypothesis, this finding was expected. However, analysis of protein expression using IHC of canonical transcription factors interestingly showed higher GATA3 over T-Bet and RORgT (Fig. 1j-l). (GATA3, or GATA-binding protein 3, is a Th2 transcription factor; Tbet, or $\mathrm{T}$ box transcription factor, is a Th1 transcription factor; and RORgT, or retinoic acid receptor-related orphan nuclear receptor, is a Th17 transcription factor). There was also minimal FoxP3 expression, indicating a limited contribution from Tregs (Fig. 1i). Taken together, these findings suggest that ipilimumab-induced Grover's disease may result from action of Th2 cells with much less contribution from Th17. Moreover, the finding that pembrolizumab therapy did not exacerbate our patient's Grover's disease is consistent with pre-clinical data demonstrating that PD-1 inhibition induces Th1/Th17 responses while producing less Th2 type responses [14].

In light of our patient's clinical history with supportive histologic and immunologic data, we believe that ipilimumab-induced Grover's disease is an irAE that may be mediated by specific up-regulation and infiltration of Th2 rather than Th17 cells. This aligns with a previous report implicating IL-4, a known Th2 inducer, as a causative factor for Grover's disease [7]. Having a deeper understanding of the characteristic immune response that mediates irAEs, and specifically dermatological irAEs in relation to Grover's disease, may have a tremendous impact on how we manage and prevent them in a targeted way. As proof of concept, 
recent data has been published demonstrating the efficacy of IL-17A inhibition in the treatment of psoriatic arthritis [15]. Additionally, IL-6 receptor inhibitors are FDA approved for autoimmune disorders like rheumatoid arthritis and Crohn's disease. This targeted approach to immune suppression through selective inhibition of autoreactive cells like Th2/Th17 while minimally affecting the tumor specific Th1/CD8 cells, may have better efficacy in treating irAEs from CPI than generalized immune suppression with corticosteroids, which is the current practice. Furthermore, such an approach may preserve the cytotoxic effect of CPI. Obviously, this hypothesis can be confirmed only through prospective, randomized trials. Our report also illustrates the increasing complexity and wide variety of immune toxicities not experienced with traditional therapies.

\section{Conclusion}

In conclusion, we report an addition case of Grover's disease induced by ipilimumab. Interestingly, this dermatologic side effect did not appear with use of anti-PD-1 therapy in our patient. Although its exact pathogenesis is unknown, our immune analysis suggests a possible role of Th2 cells, which may warrant additional study.

\section{Abbreviations}

CPI, checkpoint inhibitor; CT, computed tomography; CTLA-4, cytotoxic T-lymphocyte-associated protein 4; FDA, US Food and Drug Administration; $I H C$, immunohistochemistry; IL, interleukin; irAE, immune related adverse event; IV, intravenous; PD-1, programmed cell death protein 1

\section{Acknowledgements}

All authors report no relevant conflicts of interest. The authors wish to thank Ms. Kim Vu, Department of Pathology at MDACC for her excellent assistance in preparing the figures.

\section{Funding}

None.

\section{Availability of data and materials}

Not applicable.

\section{Authors' contribution}

MU, FF and AD wrote and edited the final manuscript; $C H, C B, J L C$ and MTT performed all immunologic analysis, prepared pathology figures, and interpreted pathology tissue; NM, ES and LB coordinated the patient's care in our melanoma clinic; $\mathrm{CWH}$ helped prepare relevant pathology tissue for analysis; PH provided intellectual guidance on immune studies and paper concept. All authors read and approved the final manuscript.

\section{Competing interests}

The authors declare that they have no competing interests.

\section{Consent for publication}

All authors give their consent for publication.

\section{Ethical approval and consent to participate}

The patient upon whom this case is focused has given his informed consent/consent to publish on the clinical details that are contained within this report. The peripheral blood used in this study was collected and analyzed under IRB-approved protocols (IRB LAB00-063; LAB03-0320; 2012-0846).

\section{Author details}

${ }^{1}$ Department of Melanoma Medical Oncology, University of Texas-MD Anderson Cancer Center, Houston, TX, USA. ²Department of Pathology, University of Texas-MD Anderson Cancer Center, Houston, TX, USA.

Received: 1 April 2016 Accepted: 4 August 2016

Published online: 20 September 2016

\section{References}

1. Grover RW. Transient acantholytic dermatosis. Arch Dermatol. 1970;101(4):426-34

2. Chalet M, Grover R, Ackerman AB. Transient acantholytic dermatosis: a reevaluation. Arch Dermatol. 1977;113(4):431-5.

3. Davis MD, Dinneen AM, Landa N, Gibson LE. Grover's disease: clinicopathologic review of 72 cases. Mayo Clin Proc. 1999;74(3):229-34.

4. Guana AL, Cohen PR. Transient acantholytic dermatosis in oncology patients. J Clin Oncol. 1994;12(8):1703-9.

5. Anforth R, Fernandez-Penas P, Long GV. Cutaneous toxicities of RAF inhibitors. Lancet Oncol. 2013;14(1):e11-18.

6. Moon HR, Lee JH, Rhee DY, Won CH, Chang SE, Lee MW, Choi JH, Moon KC. Grover's Disease in a Liver Transplant Patient. Ann Dermatol. 2014;26(1):117-8.

7. Mahler SJ, De Villez RL, Pulitzer DR. Transient acantholytic dermatosis induced by recombinant human interleukin 4. J Am Acad Dermatol. 1993;29(2 Pt 1):206-9.

8. Munoz J, Guillot B, Girard C, Dereure O, Du-Thanh A. First report of ipilimumab-induced Grover disease. Br J Dermatol. 2014;171(5):1236-7.

9. Hodi FS, O'Day SJ, McDermott DF, Weber RW, Sosman JA, Haanen JB, Gonzalez R, Robert C, Schadendorf D, Hassel JC, et al. Improved survival with ipilimumab in patients with metastatic melanoma. N Engl J Med. 2010;363(8):711-23.

10. Robert C, Thomas L, Bondarenko I, O'Day S, Weber J, Garbe C, Lebbe C, Baurain JF, Testori A, Grob JJ, et al. Ipilimumab plus dacarbazine for previously untreated metastatic melanoma. N Engl J Med. 2011;364(26):2517-26.

11. Chambers CA, Sullivan TJ, Allison JP. Lymphoproliferation in CTLA-4deficient mice is mediated by costimulation-dependent activation of CD4+ T cells. Immunity. 1997;7(6):885-95.

12. Callahan MK, Wolchok JD, Allison JP. Anti-CTLA-4 antibody therapy: immune monitoring during clinical development of a novel immunotherapy. Semin Oncol. 2010;37(5):473-84.

13. Callahan MK, Yang A, Tandon S, Xu Y, Subudhi SK, Roman RA, Heine Al, Pogoriler E, Kuk D, Panageas K et al. Evaluation of serum IL-17 levels during ipilimumab therapy: Correlation with colitis. J Clin Oncol. 29:2011 (suppl; abstr 2505).

14. Dulos J, Carven GJ, van Boxtel SJ, Evers S, Driessen-Engels LJ, Hobo W, Gorecka MA, de Haan AF, Mulders P, Punt CJ, et al. PD-1 blockade augments Th1 and Th17 and suppresses Th2 responses in peripheral blood from patients with prostate and advanced melanoma cancer. J Immunother. 2012;35(2):169-78.

15. Mease PJ, Mclnnes IB, Kirkham B, Kavanaugh A, Rahman P, van der Heijde D, Landewe R, Nash P, Pricop L, Yuan J, et al. Secukinumab Inhibition of Interleukin-17A in Patients with Psoriatic Arthritis. N Engl J Med. 2015;373(14):1329-39.

Submit your next manuscript to BioMed Central and we will help you at every step:

- We accept pre-submission inquiries

- Our selector tool helps you to find the most relevant journal

- We provide round the clock customer support

- Convenient online submission

- Thorough peer review

- Inclusion in PubMed and all major indexing services

- Maximum visibility for your research

Submit your manuscript at www.biomedcentral.com/submit 